\title{
Eosinophilic Fasciitis: an Atypical Presentation of a Rare Disease
}

\author{
Sabrina Poradosúa, Esther Vanderlinden ${ }^{b}$, Alex Michottec, Hendrik De Raeve ${ }^{d}$, Sabine D. Allard ${ }^{a}$ \\ a Department of Internal Medicine, Universitair Ziekenhuis Brussel, Vrije Universiteit Brussel, Brussels, Belgium \\ bDepartment of Pathology, Algemeen Stedelijk Ziekenhuis, Aalst, Belgium
}

'Department of Neurology and Pathology (Neuropathology), Universitair Ziekenhuis Brussel, Vrije Universiteit Brussel, Brussels, Belgium

${ }^{\mathrm{d} D e p a r t m e n t}$ of Pathology, Universitair Ziekenhuis Brussel, Vrije Universiteit Brussel, Brussels, Belgium

Received: 30/01/2015

Accepted: 09/02/2015

Published: 03/04/2015

How to cite this article: Poradosú S, Vanderlinden E, Michotte A, De Raeve H, Allard SD. Eosinophilic fasciitis: an atypical presentation of a rare disease. EJCRIM 2015;2:doi: 10.12890/2015_000186

Conflicts of Interests: The authors declare that they have no conflicts of interest in this research

This article is licensed under a Commons Attribution Non-Commercial 4.0 License

\section{ABSTRACT}

Objectives: We report an atypical presentation of eosinophilic fasciitis and provide a concise overview of the literature.

Materials and Methods: Clinical and laboratory findings in a patient presenting with fever and skin induration were recorded. A deep muscle biopsy was performed in order to confirm the diagnosis.

Results: A spontaneous favourable clinical and radiological evolution was observed.

Conclusion: The diagnosis of eosinophilic fasciitis is challenging due to the lack of pathognomonic signs and symptoms. As spontaneous resolution has been described, watchful waiting is defendable depending on the clinical presentation. Although magnetic resonance imaging (MRI) can be useful in establishing the diagnosis, a deep muscle biopsy remains the gold standard diagnostic tool.

\section{LEARNING POINTS}

- Eosinophilic fasciitis may spontaneously evolve favourably.

- Eosinophilic fasciitis should be considered in the differential diagnosis of fever associated with skin and soft tissue inflammation.

- The diagnosis of eosinophilic fasciitis is often challenging due to the lack of pathognomonic signs and symptoms.

\section{KEYWORDS}

Eosinophilic fasciitis, Shulman's disease, eosinophilia, oedema

\section{INTRODUCTION}

Eosinophilic fasciitis or Shulman's disease is an uncommon connective tissue disease characterized by inflammation, swelling and thickening of the skin and fascia of the limbs and/or trunk, peripheral eosinophilia, hypergammaglobulinaemia and elevated erythrocyte sedimentation rate $^{[1]}$. Only a few hundred cases have been reported since it was first described by Schulman in $1974^{[2]}$. It can affect people of all ages and of both genders, being more common between the ages of 30 and 60 years old. Although the underlying aetiology is unknown, possible triggers are excessive physical activity, trauma and medication ${ }^{[1]}$. Associated autoimmune, infectious, oncological and haematological diseases and an aberrant immune response have also been described. We report a case of eosinophilic fasciitis in a patient with no underlying condition.

\section{CASE REPORT}

A 23-year-old man was referred to our Internal Medicine Department with fever, general weakness and painful skin indurations distributed asymmetrically over his lower limbs. The patient mentioned a healthy lifestyle with regular sport activities, no alcohol, drug or tobacco abuse and no recent travel history or close animal contact.

Two weeks before the onset of symptoms, he complained of sore throat and fever lasting for approximately 10 days. Subsequently, he noticed a nodule on the lower left limb together with progressive swelling, redness and pain. Laboratory tests showed signs of inflammation (Table 1). 


\begin{tabular}{|c|c|c|}
\hline Variable & Value & Reference range \\
\hline Sedimentation rate $(\mathrm{mm} / \mathrm{h})$ & 30 & $<10$ \\
\hline C-reactive protein (mg/l) & 91 & $<5$ \\
\hline Sodium (mmol/l) & 142 & $136-146$ \\
\hline Potassium (mmol/l) & 4.1 & $3.5-5.3$ \\
\hline Chloride (mmol/l) & 101 & $96-111$ \\
\hline Red blood count $\left(\times 10^{6} / \mathrm{mm}^{3}\right)$ & 5.01 & $4.63-6.08$ \\
\hline Haematocrit (\%) & 43.6 & $40.1-51.0$ \\
\hline Haemoglobin (g/dl) & 14.7 & $13.7-17.5$ \\
\hline White cell count $\left(/ \mathrm{mm}^{3}\right)$ & 7980 & $4230-9070$ \\
\hline \multicolumn{3}{|l|}{ Differential count (\%) } \\
\hline Neutrophils & 82 & $41-74$ \\
\hline Eosinophils & 2 & $1-7$ \\
\hline Basophils & 0 & $0-1$ \\
\hline Lymphocytes & 8 & $22-53$ \\
\hline Monocytes & 8 & $5-12$ \\
\hline Thrombocytes $\left(\times 10^{3} / \mathrm{mm}^{3}\right)$ & 152 & $140-337$ \\
\hline Iron ( $\mu \mathrm{g} / \mathrm{dl})$ & 52 & $59-158$ \\
\hline Fibrinogen (mg/dl) & 704 & $150-400$ \\
\hline D-dimer (ng/ml) & 665 & $68-494$ \\
\hline Partial thromboplastin time (s) & 26.9 & $24.5-32.8$ \\
\hline Prothrombin time (I.N.R.) & 1 & $2.0-4.5$ \\
\hline
\end{tabular}

Table 1: Laboratory results at onset of complains

An ultrasound of the lower left extremity showed two irregularly delineated hyperechogenic muscular regions located in the left gastrocnemius ( $44 \mathrm{~mm}$ diameter) and left soleus muscle ( $26 \mathrm{~mm}$ diameter). These findings were suggestive of a myositis for which amoxicillin/ clavulanic acid $875 \mathrm{mg}$ TID was empirically started by his general practitioner with only transient clinical improvement. The patient was therefore referred to a peripheral hospital. A new ultrasound of his lower left limb was unchanged. Chest X-ray, echocardiography, fundoscopy and ultrasound of the abdomen were normal. It was decided to switch empirically from oral to intravenous amoxicillin/clavulanic acid $(4 \times 1 \mathrm{~g} /$ day) and to transfer him to our department.

On the day of admission, the patient was apyretic; blood pressure and heart rate were 107/68 mmHg and 88/min, respectively. Oxygen saturation was normal. Physical examination showed a warm and erythematous left leg with two painful skin indurations: one located medial to the tibial bone and the other at the calf. The skin around the right knee was erythematous and warm, with the presence of a subcutaneous nodule resulting in functional impairment of the knee. The remainder of the physical examination was normal. Laboratory findings showed persistent signs of inflammation (Table 2). Empiric treatment with amoxicillin/clavulanic acid was continued.

On Day 3 of admission, the patient's condition worsened: he developed fever $\left(>38.5^{\circ} \mathrm{C}\right.$ ), myalgia, pain in the left forearm and sore throat. The inflammation parameters increased. Serology and humoral immunology tests remained negative. We started a therapeutic window and performed a fluorodeoxyglucose positron emission tomography/computed tomography (FDG PET/CT) scan and a magnetic resonance image (MRI) of the lower limbs, which confirmed the presence of multiple hypermetabolic muscular foci (Fig. 1) and a possible bilateral multifocal myositis (Fig. 2).

However, an electromyogram could not confirm the presence of a bilateral myositis.

On Day 5 of admission and 48h after interruption of the empiric antibiotics, a diagnostic deep skin and muscle biopsy was performed. The biopsy showed an inflammatory infiltrate in the fascia consisting largely of eosinophils (Fig. 3), compatible with an eosinophilic fasciitis. Aerobic and anaerobic cultures of the muscle samples remained negative.

Over the next days, the patient showed a spontaneous favourable clinical and biochemical evolution. Therefore, no immunosuppressive regimen was prescribed. The patient was discharged on Day 14 after admission. Four weeks later, we performed an MRI of the lower limbs (Fig. 4), showing a spontaneous resolution of the inflammation. 


\begin{tabular}{|c|c|c|}
\hline Variable & On admission & Reference range \\
\hline C-reactive protein (mg/l) & 131 & $<5$ \\
\hline Creatinine phosphokinase (U/I) & 247 & $<145$ \\
\hline Sodium (mmol/l) & 145 & $137-145$ \\
\hline Potassium (mmol/l) & 4.2 & $3.6-5.0$ \\
\hline Chloride (mmol/l) & 103 & $101-111$ \\
\hline Red blood count $\left(\times 10^{6} / \mathrm{mm}^{3}\right)$ & 4.9 & $4.2-5.7$ \\
\hline Haematocrit (\%) & 43.4 & $39.6-49.2$ \\
\hline Haemoglobin (g/dl) & 14.6 & $13-16.5$ \\
\hline White cell count (per $\mathrm{mm}^{3}$ ) & 11500 & $3600-9600$ \\
\hline \multicolumn{3}{|l|}{ Differential count (\%) } \\
\hline Neutrophils & 81.9 & $41-74$ \\
\hline Eosinophils & 1.8 & $0-6$ \\
\hline Basophils & 0.2 & $0-2$ \\
\hline Lymphocytes & 9.4 & $19-44$ \\
\hline Monocytes & 6.7 & $3-13$ \\
\hline Thrombocytes $\left(\times 10^{3} / \mathrm{mm}^{3}\right)$ & 187 & $158-450$ \\
\hline Fibrinogen (mg/dl) & 827 & $180-400$ \\
\hline Partial thromboplastin time (s) & 26.7 & $22.2-34.4$ \\
\hline Prothrombin time (I.N.R.) & 1.1 & $0.8-1.3$ \\
\hline
\end{tabular}
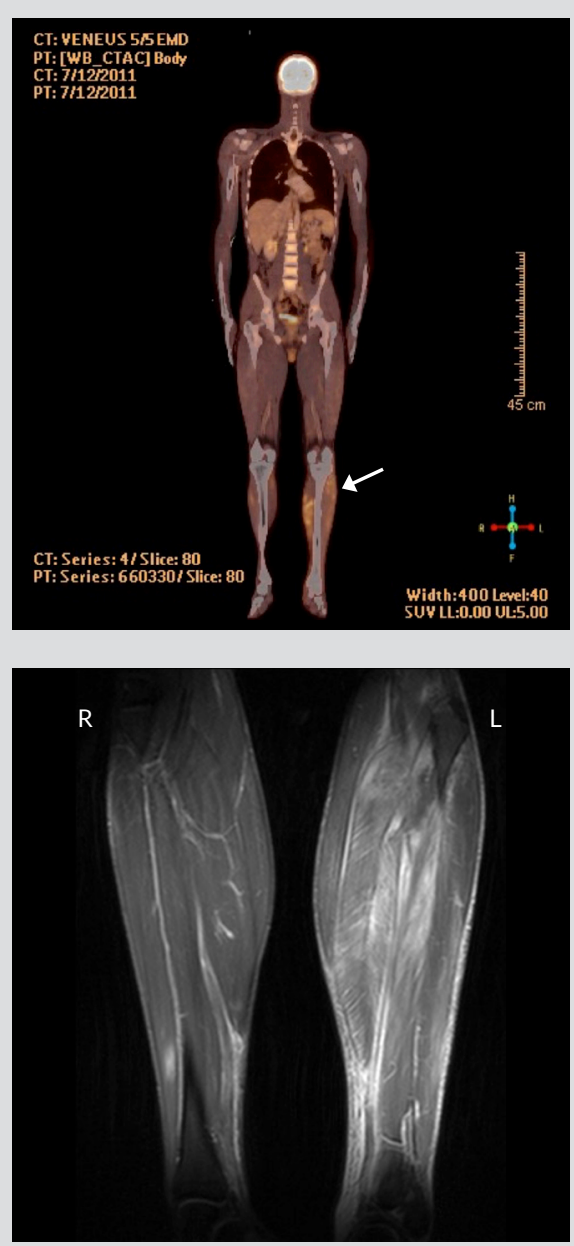
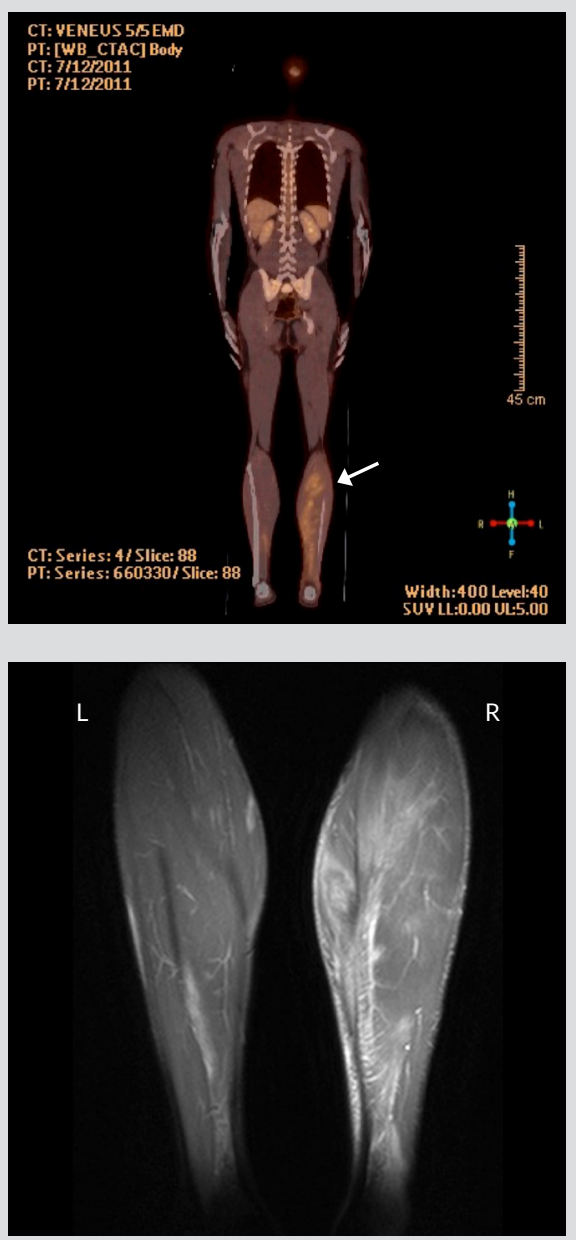

Figure 1: FDG PET/CT scan shows multiple FDG-positive foci in both lower extremities (white arrows). There were no zones of liquefaction. The presence of reactive lymph nodes and bone marrow stimulation of the axial skeleton and pelvic girdle can also be seen. Right panel: anterior view. Left panel: posterior view.

Figure 2: $T_{2}$-weighted MRI of the lower extremities. Right panel: fusiform hypersignals in the extensors and peroneal compartment, in the medial gastrocnemius and the distal flexor hallucis. Left panel: hypersignal with extra enhancement of the tibialis anterior, extensor digitorum, tibialis posterior, soleus and gastrocnemius muscles, compatible with myositis. The presence of subcutaneous oedema, especially at the left inferior extremity, can also be seen. There is no indication of abscedation. 

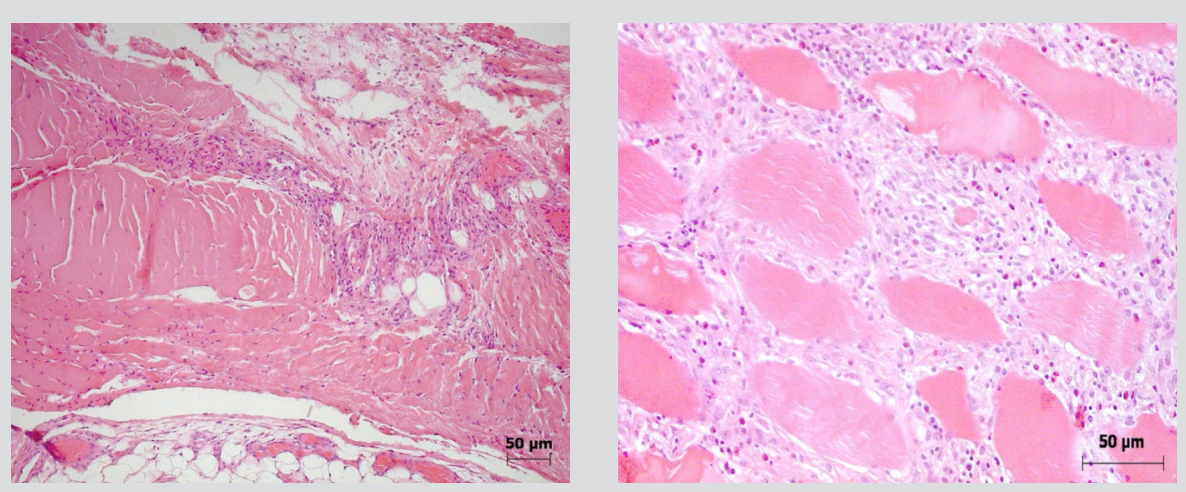

Figure 3: Muscle biopsy (haematoxylin and eosin stain). (A) Section through muscle and hypodermis showing visualization of inflammatory infiltration of the fascia. (B) $\times 200$ enlargement showing that the inflammatory infiltrate consisting of lymphocytes and polynuclear eosinophils is limited to the fascia, with no muscular involvement.

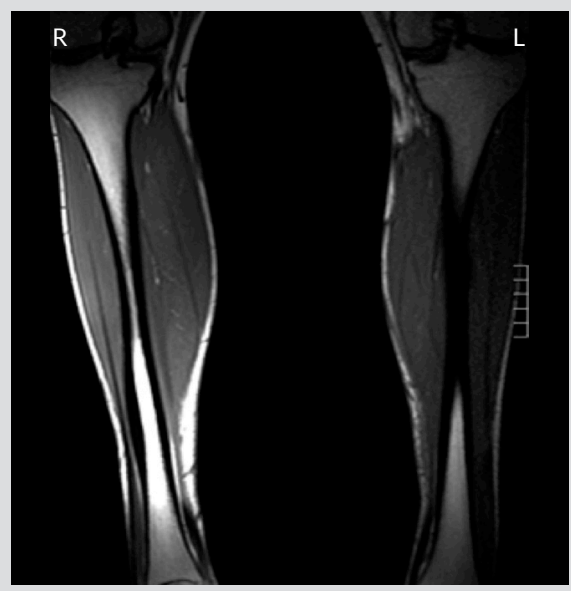

Figure 4: $T_{1}$-weighted MRI of the lower extremities one month after discharge. Compared to the previous MRI, there is a reduction of the signal intensity of the right medial gastrocnemius muscle, the distal soleus muscle, the extensor longus muscle and the flexor longus muscle. There is no abscedation, bone marrow oedema or periosteal reaction.

After a period of 3 years, the patient has experienced occasional pain in the lower limbs, mainly following physical activities, but without associated fever, skin lesions or inflammatory blood parameters.

\section{DISCUSSION}

Eosinophilic fasciitis usually presents with symmetric erythema of the extremities and symmetric subcutaneous indurations, which subsequently evolve into 'peau d'orange' and non-pitting oedema. This is in contrast with the clinical presentation of our patient. Nonspecific systemic symptoms are often present. Eosinophilic fasciitis presenting with Raynaud's phenomenon, CREST syndrome or visceral infiltration is extremely rare and, if present, is more likely to be associated with a systemic scleroderma or another systemic syndrome than with eosinophilic fasciitis. Skin of the face, hands and feet is usually not affected. The groove sign is a common feature that can help distinguish eosinophilic fasciitis from systemic sclerosis, but is non-specific, as it has also been described in other conditions ${ }^{[3]}$.

Although peripheral eosinophilia is the most common laboratory finding in eosinophilic fasciitis, it may not always be present, as was the case in our patient. Eosinophilia is usually mild, making it a poor diagnostic tool and a weak marker of disease activity ${ }^{[1]}$. However, eosinophilic fasciitis has also been associated with primary eosinophilia ${ }^{[4]}$.

Other laboratory findings may include polyclonal hypergammaglobulinaemia and increased inflammatory markers. Autoimmune markers such as anti-nuclear antibodies might be positive in low titres ${ }^{[1]}$ and muscle enzymes are not necessarily elevated. In our patient, there was no hypergammaglobulinaemia and the creatine phosphokinases were slightly elevated. MRI plays an important role in the diagnosis of eosinophilic fasciitis and in the follow-up ${ }^{[5]}$. An FDG-PET scan may provide information about fascial changes and help to exclude an underlying malignancy.

A deep muscle biopsy is, however, the cornerstone for diagnosis. Histologic findings usually include a normal epidermis; subcutaneous infiltration by lymphocytes, eosinophils, plasma cells and histiocytes; and a thickened fascia with sclerosis and inflammatory polymorphic infiltrates with varying numbers of eosinophils. In some cases, an eosinophilic perimyositis or infiltration of the muscle has been observed. The inflammatory infiltrates seen in our patient consisted largely of eosinophils in the fascia, spreading to the adjacent muscle. This valuable histological information combined with the clinical and radiological presentation contributed to the diagnosis of eosinophilic fasciitis.

The main differential diagnoses include infections, neoplasia, systemic diseases, tumour-like lesions, toxic oil syndrome and eosinophiliamyalgia syndrome ${ }^{[6]}$. In our patient, no underlying disease was diagnosed.

First-line treatment consists of high-dose corticosteroids followed by slow tapering. Relapses may occur, and patients may not respond to glucocorticoids. In these patients, the use of other immunosuppressive agents, including methotrexate, hydroxychloroquine and others, might be necessary. The prognosis is usually favourable, as complete remission is generally achieved, even if prolonged or combined immunosuppressive treatment is necessary ${ }^{[7]}$. The absence of clinical response should prompt further investigation to rule out an underlying malignancy. 
No corticosteroids were initiated in our patient as progressive spontaneous remission was observed. Since its first description by Schulman in $1974^{[2]}$, only a few hundred cases of eosinophilic fasciitis have been reported worldwide; spontaneous remission has been observed in about $10-20 \%$ of patients ${ }^{[8]}$.

As eosinophilic fasciitis might relapse and precede the onset of a haematologic malignancy, close monitoring of patients is indicated, even after interruption of the treatment.

\section{REFERENCES}

1. Pinal-Fernandez I, Selva-O'Callaghan A, Grau JM. Diagnosis and classification of eosinophilic fasciitis. Autoimmun Rev 2014;13:379-382.

Shulman LE. Diffuse fasciitis with eosinophilia: a new syndrome? Trans Assoc Am Physicians 1975;88:70-86.

Nair PS, Nanda KG, Jayapalan S. The "sign of groove", a new cutaneous sign of internal malignancy. Indian J Dermatol Venereol Leprol 2007;73:141.

Guillevin L, Meyer O, Sibilia J. Traité des Maladies et Syndromes Systémiques, 5th ed. Parijs. Médecine Sciences Publications, 2008.

Ronneberger MR, Janka R, Schett G, Manger B. Can MRI substitute for biopsy in eosinophilic fasciitis? Ann Rheum Dis 2009;68:1651-1652.

6. Helfgott SM, Varga J. Eosinophilic fasciitis. UpToDate. Wolters Kluwer. http://www.uptodate.com/contents/eosinophilic-fasciitis?source=search_result\&search=eosinophilic+fasciitis\&selectedTitle=1 \%7E21. Last accessed 01/12/2014

7. Boin F, Hummers LK. Scleroderma-like fibrosing disorders. Rheum Dis Clin North Am 2008;34:199-220

8. Islam MN, Islam MA, Abdal SJ, Azad MAK, Ahmedullah AK, Haq SA. Eosinophilic fasciitis: what matters in management in a developing country-a case report with two and a half-year follow-up. J Health Popul Nutr 2012;30:117-120. 